\title{
CLINICO-RADIOLOGICAL RESULTS OF HEMIEPIPHYSIODESIS USING EIGHT-PLATE IN ADOLESCENTS AND PAEDIATRIC AGE GROUPS WITH IDIOPATHIC BILATERAL GENU VALGUM
}

\author{
Mozammil Pheroz ${ }^{1}$, Arvind Kumar², Ankit Jain ${ }^{3}$, Siddharth Gupta ${ }^{4}$, Rajesh K. Chopra ${ }^{5}$, Narendra Kumar 6 , Kumar Keshav ${ }^{7}$
}

${ }^{1}$ Senior Resident, Department of Orthopaedics, CIO, VMMC and Safdarjung Hospital, New Delhi. India.

${ }^{2}$ Senior Resident, Department of Orthopaedics, CIO, VMMC and Safdarjung Hospital, New Delhi. India.

${ }^{3}$ Senior Resident, Department of Orthopaedics, CIO, VMMC and Safdarjung Hospital, New Delhi. India.

${ }^{4} J u n i o r$ Resident, Department of Orthopaedics, CIO, VMMC and Safdarjung Hospital, New Delhi. India.

${ }^{5}$ Director Professor, Department of Orthopaedics, CIO, VMMC and Safdarjung Hospital, New Delhi. India.

${ }^{6}$ Professor, Department of Orthopaedics, CIO, VMMC and Safdarjung Hospital, New Delhi. India.

${ }^{7}$ Senior Resident, Department of Orthopaedics, CIO, VMMC and Safdarjung Hospital, New Delhi. India.

\section{BACKGROUND}

ABSTRACT

Through this study we want to evaluate the results of hemiepiphysiodesis of distal femoral physis with eight-plate for correcting the knock knee in adolescent age group. Genu valgum is considered physiological up to the age of 3-4 years. The maximum value of genu valgum is observed at age of 3-4 years and this further spontaneously resolves around 7 years of age. Persistence of genu valgum beyond this age is pathological and requires some form of treatment.

\section{METHODS}

This is a prospective longitudinal study in which 30 patients with idiopathic bilateral genu valgum were treated surgically by hemiepiphysiodesis using eight- plates. Patient evaluated with radiograph and clinically assessed at every follow up till 36 months.

\section{RESULTS}

We evaluated 30 patients who were surgically managed for bilateral idiopathic genu valgum over a 36-month period. The average age at surgery was 13 years and 7 months (Range 11 years 8 months-14 years 6 months). The mean preoperative tibiofemoral angle was found to be $17^{0}$ with a standard deviation $+/-2.7^{0}$, and post-surgery the mean tibiofemoral angle was recorded to be $5^{0}$ with a standard deviation $+/-1^{0}$. The total follow-up period was 36 months.

\section{CONCLUSIONS}

Hemiepiphysiodesis using eight plate is a simple technique for correction of idiopathic genu valgum in adolescent and paediatric patients. It produces reproducible results, is cost effective and ensures rapid return to preoperative level of school and recreational activities.

\section{KEY WORDS}

Genu Valgum, Hemiepiphysiodesis, Eight-Plate, Tibiofemoral Angle

HOW TO CITE THIS ARTICLE: Pheroz M, Kumar A, Jain A, et al. Clinico-radiological results of hemiepiphysiodesis using eight-plate in adolescents and paediatric age groups with idiopathic bilateral genu valgum. J. Evolution Med. Dent. Sci. 2019;8(27):2197-2201, DOI: $10.14260 /$ jemds/2019/481

\section{BACKGROUND}

Genu valgum is considered physiological up to the age of 3-4 years. The maximum value of genu valgum is observed at age of 3-4 years and this further spontaneously resolves around 7 years of age. Persistence of genu valgum beyond this age is pathological and requires some form of treatment. Genu valgum may cause anterior knee pain, patellofemoral instability, circumduction gait, and difficulty running. The normal tibiofemoral angle, is the angle defined as the angle made between the mechanical axis of the femur intersecting with the mechanical axis of the tibia. ${ }^{1,2}$ The value of normal TFA is 5-70 in adolescent population. Spectrum for management of idiopathic genu valgum in adolescents and

'Financial or Other Competing Interest': None.

Submission 18-05-2019, Peer Review 20-06-2019,

Acceptance 27-06-2019, Published 08-07-2019.

Corresponding Author:

Mozammil Pheroz,

Resident Doctor's Hostel,

Safdarjung Hospital Campus,

New Delhi-110029, India.

E-mail: muzammilphrz@gmail.com

DOI: $10.14260 /$ jemds $/ 2019 / 481$ paediatric age group is very wide. Recently growth modulation using various techniques has emerged as attractive option and is considered in all patients having remaining growth potential as first line treatment. Once the physis is closed and skeletal maturity is achieved, osteotomy replaces epiphysiodesis as the treatment of choice.

Haas in 1945 reported halting the physical growth by introducing nails through it. He did his studies over animals and later reproduced the results in children. Haas reported that after he removed the nail, longitudinal growth in the limbs was re-established. Thus, he suggested a new method for the treatment of dysmetria and angular deformities of the limbs. ${ }^{3}$ It was Phemister, who for the first time described the epiphysiodesis in 19934. He removed rectangular piece of bone across the physis and then reinserted the piece in exact opposite direction, thus creating a bone bridge in the physis. This bone bridge halted the growth at the operated physis. But this method of epiphysiodesis is permanent, and if the amount of growth remaining is not estimated precisely it can lead to development of deformity in follow up. In 1979, Zuege, reported that epiphysiodesis with staples was a safe technique that can produce good results in patients treated for coronal plane deformity of lower limbs 5 . He emphasized on the need 
for proper radiological and clinical evaluation of patients before surgery so that exact timing for removal of hardware can be anticipated. Surgical stapling over the growth plate on over-grown convex side of deformity intents to halt the growth on the area where staple are applied and hence produce a gradual correction of deformity, but staple application is also associated with complications like staple breakage and loss of corrections achieved. Boven and other researchers supported a percutaneous procedure for epiphysiodesis using image intensifier for accurate curettage of physis 6 . Though there was a percutaneous technique, but it was a permanent epiphysiodesis plus damage of popliteal nerve during the procedure is a dreaded complication. Metaizeau reported better results using percutaneous transphyseal cannulated screws, but it is questionable whether the process of epiphysiodesis was reversible or not ${ }^{7}$. Stevens developed an implant made up of titanium that had 2 holes for two cannulated screws. This technique gave better results. Angular deformity corrected at fast pace. The plate applied didn't produced any compression at the physeal region so there was no physeal arrest. Loss of fixation and implant failure were rare $^{8}$. This eight-plate is a non-locking plate that is applied extra-periosteally and fixed with two screws can be of great use to avoid the complications brought with transphyseal screw and osteotomy. We have studied the clinical and radiological results of eight-plate in 30 patients with bilateral, idiopathic knock-knee.

\section{METHODS}

Our study type was prospective interventional study on 30 patients (60 knees) attending tertiary hospital OPD with bilateral genu valgum in lower limbs fulfilling the inclusion criteria. The study protocol was approved by our Institutional ethical committee. The study duration was from May 2015 to June 2018 A total of 30 patients (60 knees) with idiopathic bilateral genu valgum were managed surgically with eightplate were observed.

\section{Inclusion Criteria}

All patients who underwent surgery were examined radiographically with anteroposterior x-rays of both the knees and included in study only if they had approximately one year of predicted growth left.

\section{Exclusion Criteria}

Patients with pathological genu valgum due to trauma, tumour, warfarin, osteochondroma were not included in the study. Deranged serum calcium or alkaline phosphatase level

\section{Materials}

All the patients were operated using implants and instruments from the same manufacturer. In our study we used Orthofix plates and instrument especially intended for paediatric deformity correction (Fig. 1 a, b.)

\section{Preoperative Preparation}

Patients satisfying the inclusion criteria, after consent given by the parents were admitted and all of them underwent clinical, radiological and laboratory investigations. Blood investigation included complete blood count, liver function tests, kidney function tests, Coagulation profile (Prothrombin time, activated partial thromboplastin time, international normalized ratio), serum calcium, phosphate, alkaline phosphatase and, vitamin D level. If any of the parameters especially the biochemical parameters were deranged, surgery was held, and patient waited till all parameters came to normal. X ray scanograms (Fig. 3) were analysed for preoperative planing, the tibiofemoral angles were recorded. Clinical examinations done with special emphasis on intermalleolar distance (Fig. 2). Best possible care was taken to measure the exact intermalleolar distance. The measurements were recorded meticulously.

\section{Procedure}

All the surgeries were done by the same surgeons with implants and instruments from same manufacturers to maintain the uniformity in procedure and results. The surgery was performed under general anaesthesia with the patient positioned supine over radiolucent operating table. Single eight-plate was used per physis. The eight plates were recontoured every time in the desired shape using a bender to fit the shape of physis targeted for epiphysiodesis. Under image intensifier guidance physis is viewed and localised with $1.6 \mathrm{~mm}$ pin. A contoured eight plate was fixed over the pin using drill-guide and guide wires were put. Care was taken not to damage the physis. Once accurate placement of guide wire was achieved, drill was done to a depth of $5 \mathrm{~mm}$. First, the epiphyseal hole was drilled followed by metaphyseal. Selftapping screws were used for fixation of plates. Screws were tightened in alternating manner till the screws were fully merged with plate and plate was flushed with the bone. During the whole procedure $\mathrm{C}$ arm was used to achieve exact placement of plates as planned. Post-operative management: As no bone had been cut around and incision on medial aspect only of about 3-4 cm, patient doesn't require any immobilization in post-operative period. Patients were discharged on the following day having safely mobilised partially weight-bearing on crutches. The compression bandage was reduced after three or four days and knee motion encouraged. Full weight-bearing was usually achieved in the second week. Every 3 months the deformity correction was assessed using clinical parameters like inter-malleolar distance and photographic records kept. Radiological improvements in deformity were assessed with anteroposterior x-ray taken every three months. Once the deformity was corrected and neutral axis was achieved, hardware removal was done. Periodic follow-up every 3 months done till skeletal maturity or 36 months which ever was earlier.

\section{Statistical Evaluation}

Categorical variables were presented in number and percentage (\%) and continuous variables were presented as mean \pm SD and median. Normality of data was tested by Kolmogorov-Smirnov test. If the normality was rejected, then non parametric test was used.

\begin{tabular}{|c|c|c|}
\hline Clinical Outcomes & Number of Patients (n) & Percentage (\%) \\
\hline $\begin{array}{c}\text { Total correction of } \\
\text { deformity }\end{array}$ & 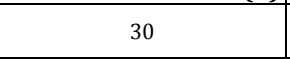 & 100 \\
\hline $\begin{array}{c}\text { Partial correction of } \\
\text { deformity }\end{array}$ & 00 & 00 \\
\hline Deformity not corrected & 00 & 00 \\
\hline $\begin{array}{c}\text { Total } \\
\end{array}$ & 30 & 100 \\
\hline
\end{tabular}




\begin{tabular}{|c|c|c|}
\hline Characteristics & Mean Value & Range \\
\hline Age & 13 years 7 months & $\begin{array}{c}11 \text { years 8 months- } \\
14 \text { years 6 months }\end{array}$ \\
\hline Duration of treatment & 12 months & 3 months-16 months \\
\hline $\begin{array}{c}\text { Tibiofemoral Angle in Preoperative } \\
\text { examination }\end{array}$ & $17^{0} \mathrm{SD}+/-2.7^{0}$ & $12^{0}-23^{0}$ \\
\hline Angle Correction achieved & $8^{0} \mathrm{SD}+/-2^{0}$ & $5^{0}-10^{0}$ \\
\hline Tibiofemoral Angle at plate removal & $5^{0} \mathrm{SD}+/-1.8^{0}$ & $4^{0}-6^{0}$ \\
\hline Degree of correction per month & $0.9^{0} \mathrm{SD}+/-0.7^{0}$ & $0.6^{0}-0.7^{0}$ \\
\hline \multicolumn{2}{|c|}{ Table 2. Clinical Findings in Patients in The Study } \\
\hline SD = Standard deviation; ${ }^{0}=$ degree \\
\hline
\end{tabular}
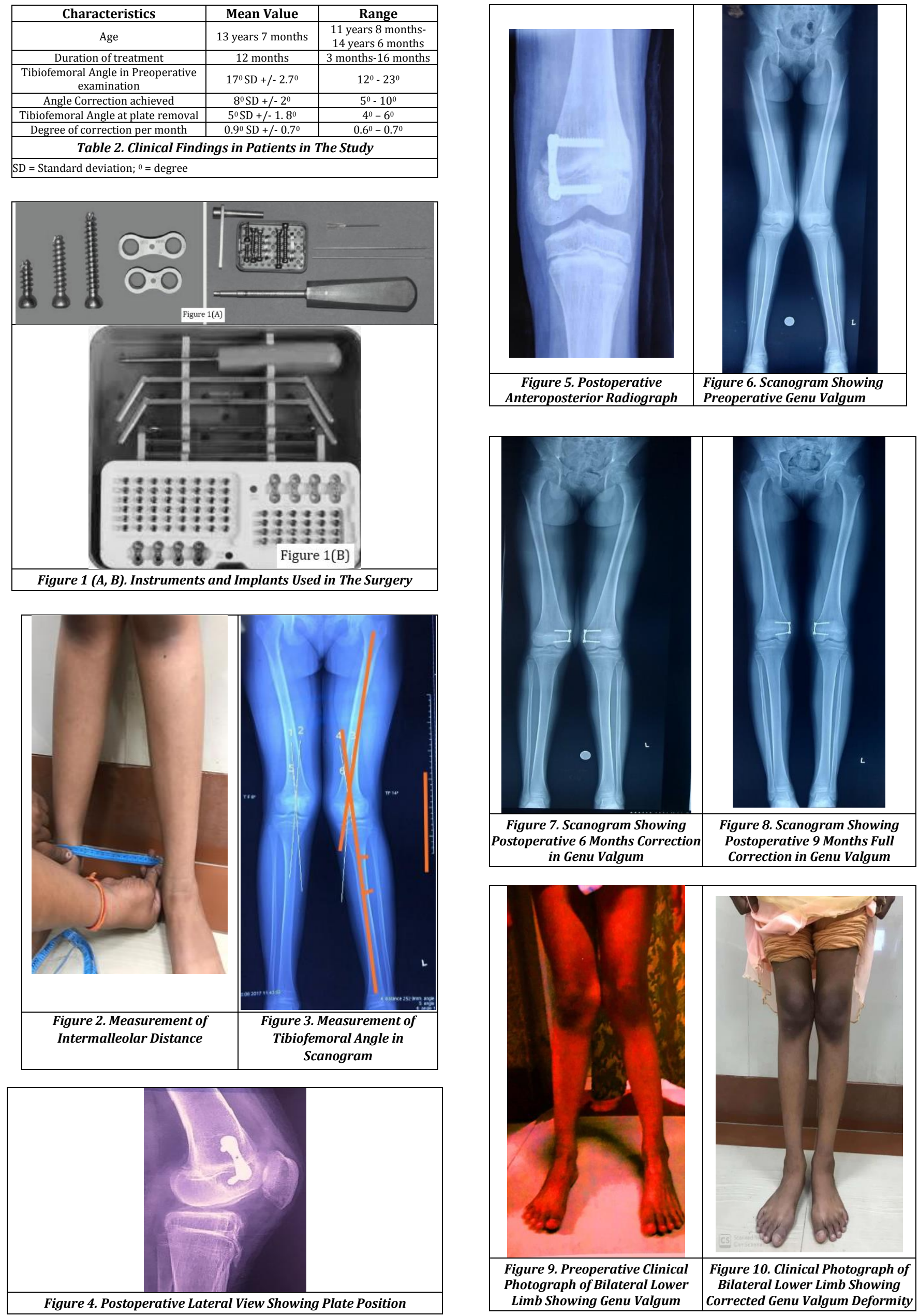
Statistical tests were applied as follows-

1. Quantitative variables were compared using unpaired ttest/Mann-Whitney Test (when the data sets were not normally distributed) between the two groups.

2. Qualitative variables were correlated using Chi-Square test/Fisher's exact test.

A p value of $<0.05$ was considered statistically significant. The data was entered in MS EXCEL spreadsheet and analysis was done using Statistical Package for Social Sciences (SPSS) version 21.0.

\section{RESULTS}

A total of 30 patients comprised the study population, and 60 knees were surgically treated for idiopathic genu valgum over a 36 months interval. The mean age at implantation was 13 year and 7 months with a range of 11 years 8 months to 14 years 6 months. The mean duration of treatment was 12 months with a range between 3 months to 16 months. Mean follow-up after surgery was 3 years. None of the patients required physical therapy, and full functional recovery was achieved post-surgery in all patients. All of the patient participating in the study achieved full correction of deformity at knee. The clinical and radiological preoperative evaluation was carried out with the patient in the standing position, during which time the tibiofemoral angle was measured in order to assess the degree of genu valgum (Table 1,2 ). Tibiofemoral angle in preoperative examination was found to be $17^{\circ} \mathrm{SD}+/-2.7^{\circ}$ with range $12^{\circ}$ to $23^{\circ}$. Angle corrections achieved was $8^{\circ} \mathrm{SD}+/-2^{\circ}$ with range $5^{\circ}$ to $10^{\circ}$. Tibiofemoral angle at plate removal was $5^{\circ} \mathrm{SD}+/-1.8^{\circ}$ with range $4^{\circ}$ to $6^{\circ}$. Degree of correction per month was $0.9^{\circ} \mathrm{SD}+/{ }^{-0.7^{\circ}}$ with range $0.6^{\circ}$ to $0.7^{\text {o. }}$ During the follow-up period, none of the patients complained of pain or discomfort while walking or during school physical activities. Satisfactory results were defined as an intermalleolar distance of less than $6 \mathrm{~cm}$, and $100 \%$ of the treated physes were considered to be satisfactory in this study. No repeat surgery done. No rebound deformity occurred. No knee pain, knee effusion or septic arthritis knee observed in our study.

\section{DISCUSSION}

Knock knee patients are very common in orthopaedics outpatient department. Considering the frequency, it is obvious to have so many methods of treatment for the same. ${ }^{9}$ In adolescents or paediatrics age group where almost one year of growth potential is still there, growth modulation using hemiepiphysiodesis for correction of angular deformity has emerged as flag bearer in recent past. Coronal deformities are commonly presented in children and growth modulation using eight-plate to correct deformity is an attractive concept in skeletally immature patients. This has rapidly gained popularity, but evidence available in literature is limited and many questions remain unanswered. The evidence is based on mostly retrospective studies and an experimental study. It can be used in idiopathic as well as pathological angular deformity. Though pathological genu valgum secondary to causes like osteochondroma or hereditary multiple exostosis or infection around knee are supposed to be resistant and may take comparatively longer time for correction after surgery. This technique has very fewer complications compared to stapling or epiphyseal screws. Skeletal maturity and damaged physis leading to closure are only few contraindications to hemiepiphysiodesis using eight plates. Idiopathic genu valgum patients were treated only after it was clear that physiological recovery is further not possible. Surgery is usually not done in age less than 8 years. ${ }^{10}$ After the age of 8 years, any deformity of a magnitude of less than 10 degree is usually managed conservatively. ${ }^{10-13}$ But if there is a deformity with in more than 16 degree and if there is atleast 12 months anticipated growth remaining in the skeleton. ${ }^{8}$ plate epiphysiodesis is better option. The technique of hemiepiphysiodesis is well established in children, in particular the use of staples and transphyseal screws. Both methods exert compression on the physis and, when placed eccentrically, can retard development on the side of application and thereby produce asymmetrical growth. The 8 plate acts on the principle of flexible tension band rather than compression of physis and thus doesn't causes premature physeal closure, that slower down the speed of growth at that particular site.4,7,14

The placement of this non-rigid tension band device at the perimeter of the physis produces the effect of a focal hinge. While some compression is applied across the physis, it is not constant owing to the propensity of the screws to diverge as correction proceeds. Once the screws reach their maximum divergence, there is also the facility within the flexible plate to bend. Both of these features reduce the risk of creating a permanent physeal tether through excessive and prolonged compression across the physis. Also, only one plate per physis is needed to produce correction, whereas three staples per physis are usually needed for the same effect. Doing hemiepiphysiodesis further has secondary benefit of better alignment of body axis leading to efficient function at hip and ankle joint. 4 The rate of deformity correction with eight plate is also good. The rate of correction depends on the rate of growth at the time of implantation. This rate varies between the boys and girls with the same chronological age and depends more on the level of skeletal maturity. Using hemiepiphysiodesis, we can avoid or delay osteotomy, rebound growth or reappearance of deformity. No hardware failure. No plate breakage is observed in the study which is in congruence with author studies found in literature.4,9,15

\section{Limitation of The Study}

The study population was very small. A larger study with larger sample is needed to corroborate the results of this study.

\section{CONCLUSIONS}

Eight-plate can be a better treatment option for idiopathic genu valgum in adolescents and paediatric population. It is a simple procedure which produces reproducible results with fewer complications. It is cost effective and saves considerable time in rehabilitation as well and assures early return to school activities.

\section{REFERENCES}

[1] Sánchez M, Pedro A. Manual práctico para residentes de ortopedia. Bogotá: Editorial Carbel, 2004: p. 428-75.

[2] Bylski-Austrow DI, Wall EJ, Rupert MP, et al. Growth plate forces in the adolescent human knee: a radiographic and mechanical study of epiphyseal staples. J Pediatr Orthop 2001;21(6):817-23.

[3] Hass SL. Retardation of bone growth by a wire loop. J Bone Joint Surg (Am) 1945;27:25-36. 


\section{Jemds.com}

[4] Phemister DB. Operative arrest of longitudinal growth of bones in the treatment of deformities. J Bone Joint Surg Am 1933;15:1-15.

[5] Zuege RC, Kempken TG, Blount WP. Epiphyseal stapling for angular deformity at the knee. J Bone Joint Surg Am 1979;61(3):320-9.

[6] Bowen JR, Johnson WJ. Percutaneous epiphysiodesis. Clin Orthop Relat Res 1984;(190):170-3.

[7] Metaizeau JP, Wong-Chung J, Bertrand $\mathrm{H}$, et al. Percutaneous epiphysiodesis using transphyseal screws (PETS). J Pediatr Orthop 1998;18(3):363-9.

[8] Stevens P, Maguire M, Dales M, et al. Physeal stapling for idiopathic genu valgum. J Pediatr Orthop 1999;19(5):645-9.

[9] Blair VP 3rd, Walker SJ, Sheridan JJ, et al. Epiphysiodesis: a problem of timing. J Pediatr Orthop 1982;2(3):281-4.

[10] Paley D, Tetsworth K. Mechanical axis deviation of the lower limbs: preoperative planning of uniapical angular deformities of the tibia and femur. Clin Orthop Relat Res 1992;(280):48-64.

\section{Original Research Article}

[11] Salenius P, Vankka E. The development of the tibiofemoral angle in children. J Bone Joint Surg Am 1975;57(2):259-61.

[12] Guarniero R, Luzo CAM, Arena EC, et al. Correção de deformidade angular dos membros inferiores pela técnica de agrafagem. Geno valgo. Rev Bras Ortop 1994;29:19-23.

[13] Fraser RK, Dickens DR, Cole WG. Medial physeal stapling for primary and secondary genu valgum in late childhood and adolescence. J Bone Joint Surg $\mathrm{Br}$ 1995;77(5):733-5.

[14] Horton GA, Olney BW. Epiphysiodesis of the lower extremity: results of the percutaneous technique. J Pediatr Orthop 1996;16(2):180-2.

[15] De Brauwer V, Moens P. Temporary hemiepiphysiodesis for idiopathic genua valga in adolescents: percutaneous transphyseal screws (PETS) versus stapling. J Pediatr Orthop 2008;28(5):549-54. 